\title{
Securely Obfuscating Re-encryption
}

\author{
Susan Hohenberger ${ }^{1,2}$, Guy N. Rothblum ${ }^{3, \star}$, \\ abhi shelat ${ }^{2}$, and Vinod Vaikuntanathan ${ }^{3, \star \star}$ \\ 1 Johns Hopkins University \\ susan@cs.jhu.edu \\ ${ }^{2}$ IBM Zurich Research \\ \{sus, abs\}@zurich.ibm.com \\ ${ }^{3}$ MIT CSAIL \\ \{rothblum, vinodv\}@mit.edu
}

\begin{abstract}
We present the first positive obfuscation result for a traditional cryptographic functionality. This positive result stands in contrast to well-known negative impossibility results $\mathrm{BGI}^{+} 01$ for general obfuscation and recent negative impossibility and improbability GK05 results for obfuscation of many cryptographic functionalities.

Whereas other positive obfuscation results in the standard model apply to very simple point functions, our obfuscation result applies to the significantly more complicated and widely-used re-encryption functional$i t y$. This functionality takes a ciphertext for message $m$ encrypted under Alice's public key and transforms it into a ciphertext for the same message $m$ under Bob's public key.

To overcome impossibility results and to make our results meaningful for cryptographic functionalities, we use a new definition of obfuscation. This new definition incorporates more security-aware provisions.
\end{abstract}

\section{Introduction}

A recent line of research in theoretical cryptography aims to understand whether it is possible to obfuscate programs so that a program's code becomes unintelligible while its functionality remains unchanged. A general method for obfuscating programs would lead to the solution of many open problems in cryptography.

Unfortunately, Barak, Goldreich, Impagliazzo, Rudich, Sahai, Vadhan and Yang $\mathrm{BGI}^{+} 01$ s show that for many notions of obfuscation, a general program obfuscator does not exist-i.e., they exhibit a class of circuits which cannot be obfuscated. A subsequent work of Goldwasser and Kalai GK05] shows the impossibility and improbability of obfuscating more natural functionalities.

In spite of these negative results for general-purpose obfuscation, there are a few positive obfuscation results for simple functionalities such as point functions. A point function $I_{x}$ returns 1 on input $x$ and 0 on all other inputs. Canetti Can97. shows that under a very strong Diffie-Hellman assumption point

\footnotetext{
* Research supported by NSFgrant CNS-0430450 and NSF grant CFF-0635297.

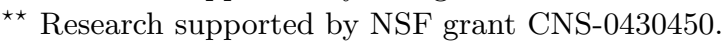


functions can be obfuscated. Further work of Canetti, Micciancio and Reingold CMR98, Wee Wee05] and Dodis and Smith DS05] relaxes the assumptions required for obfuscation and considers other (related) functionalities. Despite these positive results, obfuscators for traditional cryptographic functionalities (such as those that deal with encryption) have remained elusive.

Our Results. In this work, we present the first obfuscator for a more traditional cryptographic functionality. Namely, we show that:

Main Theorem 1 (Informal). Under reasonable bilinear complexity assumptions, there exists an efficient program obfuscator for a family of circuits implementing re-encryption.

A re-encryption program for Alice and Bob takes a ciphertext for a message $m$ encrypted under Alice's public key, and transforms it into a ciphertext for the same message $m$ under Bob's public key. Re-encryption programs have many practical applications such as the iTunes DRM system (albeit, with symmetric keys Smi05]), secure distributed file servers AFGH06 and secure email forwarding.

The straightforward method to implement re-encryption is to write a program $P$ which decrypts the input ciphertext using Alice's secret key and then encrypts the resulting message with Bob's public key. When $P$ is run by Alice, this is a good solution.

In the practical applications noted above, however, the re-encryption program is executed by a third-party. When this is the case, the straightforward implementation has serious security problems since $P$ 's code may reveal Alice's secret key to the third party. A better solution is to design an obfuscator for the re-encryption program $P$. That is, we would like that:

A third party who has a re-encryption program learns no more from the reencryption program than it does from interaction with a black-box oracle that provides the same functionality.

As we discuss later in $\oiint 1.2$, several re-encryption schemes have been proposed before BS97, BBS98, DI03, AFGH06, but none of these prior works satisfy the strong obfuscation requirement informally stated above. Our main technical contribution is the construction of a novel re-encryption scheme which meets this strong notion while remaining surprisingly practical. As a side note, in our construction, ciphertexts that are re-encrypted from Alice to Bob cannot be further re-encrypted from Bob to Carol. This may be a limitation in some scenarios, but it is nonetheless sufficient for the important practical applications noted above.

Our main conceptual contribution is a definition of obfuscation that both sidesteps impossibility results by considering randomized functionalities and is more meaningful for cryptographic applications than previous definitions of obfuscation. Let us briefly explain. 


\subsection{Notion of Secure Obfuscation}

The work of $\mathrm{BGI}^{+} 01$ views an obfuscator as a compiler which takes a program (i.e., boolean circuit) $P$ and turns it into an equivalent program that satisfies the predicate black-box property: any predicate that is computable from the obfuscated program should also be computable from black-box access to the program (see Definition 1).

Secure Obfuscation. Unfortunately, the predicate definition [BGI $\left.{ }^{+} 01\right]$ and subsequent work does not provide a meaningful security guarantee when the obfuscated program is used as part of a larger cryptographic system. Intuitively, while the predicate black-box property gives a quantifiable guarantee that some information (namely, predicates) about the program is hidden by the obfuscated circuit, other "non-black-box information" may still leak. Moreover, this leaked information might compromise the security of a cryptographic scheme which uses the obfuscated circuit. For instance, it is completely possible that an obfuscated program for "delegating signatures" both meets the predicate black-box definition and is unforgeable under black-box access to a signature oracle, yet allows an adversary who has the obfuscated program code to forge a signature!

Since many potential applications of obfuscation use obfuscated circuits in larger cryptographic schemes, the definition of obfuscation should guarantee that the security of cryptographic schemes is preserved in the following sense.

If a cryptographic scheme is "secure" when the adversary is given blackbox access to a program, then it remains "secure" when the adversary is given the obfuscated program.

The most important feature of our new definition of obfuscation is that it preserves security in the above sense, and thus we refer to it as secure obfuscation. Informally, our definition requires that if there exists a non black-box adversary with access to an obfuscated program who can break the security of a cryptographic scheme, then there exists a black-box simulator which breaks the scheme with similar probability using only black-box access to the program. Thus, if the scheme is secure against black-box adversaries, then it is also secure against adversaries with access to obfuscated programs. The definition we give in this work gives the above guarantee for any cryptographic scheme with a distinguishable attack property; any scheme where a distinguisher with public information and black-box access to the obfuscated functionality can distinguish whether or not an attacker has broken the scheme. Semantically secure encryption and re-encryption are examples of such schemes.

This new definition of obfuscation can play an important role in the design of cryptographic schemes that use obfuscation. With secure obfuscation, the design of such schemes proceeds in two stages:

1. Specify the functionality of a program (or program family), and prove security of the cryptographic scheme against an adversary given black-box access to the program.

2. Construct a secure obfuscator for the program (or program family). 
The combination of these two steps guarantees security of the scheme against an adversary that has full access to the obfuscated program. Indeed, for our scheme, we show step (1) in Theorem 3 and step (2) in Theorem 4.

Average-Case Obfuscation. Our new definition only requires obfuscation for a random circuit in a family of circuits (as in Can97, CMR98, Had00, DS05. GK05). This relaxed requirement remains meaningful for the many cryptographic applications of obfuscation in which the circuit to be obfuscated $i s$ chosen at random. Normally the random choice of a circuit corresponds to the random selection of cryptographic keys. We call the new definition of security averagecase secure obfuscation. Combining more security-aware provisions (i.e. giving a distinguisher oracle access to the functionality) with this average-case relaxation was originally suggested by Pass [Pas06].

Obfuscating Probabilistic Circuits. It is not hard to see that deterministic functionalities that are not learnable cannot be obfuscated under our definition (see \$2.2). In fact, security-preserving definitions considered in previous works $\left(\mathrm{BGI}^{+} 01\right.$, Wee05]) are only achievable for learnable deterministic functions. An important conceptual contribution of our definition and of this work is showing that these impossibility results disappear when considering obfuscation of probabilistic circuits. Furthermore, obfuscation of probabilistic circuits is important because most interesting cryptographic functionalities are probabilistic.

Other work on Obfuscation. Recently, Ostrovsky and Skeith OS05 consider a different notion of public-key obfuscation focused on keyword search. A publickey obfuscator does not maintain the functionality of a program, but rather ensures that the outputs of a public-key obfuscated program are encryptions of the original program's outputs. Adida and Wikström AW05 use a variation of this definition for public mixing. Both of these works differ from our notion of obfuscation in that our notion preserves functionality and explicitly considers black-box versus non-black-box access to a program.

\subsection{The Obfuscated Re-encryption Scheme}

Comparison with Prior Work. Mambo and Okamoto [MO97 noted the popularity of re-encryption programs in practical applications and suggested efficiency improvements over the decrypt-and-encrypt approach. Blaze, Bleumer, and Strauss introduced the notion of proxy re-encryption BS97, BBS98] in which the re-encryption program is executed by a third-party proxy. In their security notion, the proxy cannot read the messages of either Alice or Bob. The Blaze et al. construction is bidirectional (i.e., a program to translate ciphertexts from Alice to Bob can also be used to translate from Bob to Alice) and can be repeatedly applied (i.e., a ciphertext can be re-encrypted from Alice to Bob to Carol, etc.). Ateniese, Fu, Green, and Hohenberger AFGH06 presented a semantic-security style definition for proxy re-encryption and designed the first unidirectional scheme, although their scheme can only be applied once. Ateniese et al. also built a secure distributed storage system using their algorithms. 
While these prior works are secure under specialized definitions, they cannot be considered as obfuscations for re-encryption since they leak subtle non-blackbox information. On the other hand, the re-encryption definitions of Ateniese et al. AFGH06 provide some security guarantee with respect to dependent auxiliary inputs, which we will not consider in this work. For example, they show that even when Alice has the re-encryption program from Alice to Bob, Bob's semantic security still holds. (Although our definition does not require this, the scheme we present here satisfies this property.)

Overview of the Construction. We now provide intuition behind our construction of an obfuscator for re-encryption (see $\$ 4$ for the full construction). In a series of attempts, we develop a cryptosystem and an obfuscated re-encryption program which translates ciphertexts under $p k_{1}$ to ciphertexts under $p k_{2}$. Our starting point is a suitable public key cryptosystem.

Recall the semantically-secure encryption scheme due to Boneh, Boyen, and Shacham BBS04 as instantiated in a group $\mathbb{G}$ of order $q$ equipped with a bilinear map. The keys in this scheme are generated by selecting a random $h \stackrel{r}{\leftarrow} \mathbb{G}$ and $a, b \stackrel{r}{\leftarrow} \mathbb{Z}_{q}$, and setting $s k=(a, b, h)$ and $p k=\left(h^{a}, h^{b}, h\right)$. To encrypt a message $m \in \mathbb{G}$, select two random values $r, s \stackrel{r}{\leftarrow} \mathbb{Z}_{q}$ and output the ciphertext $C=\left[h^{a r}, h^{b s}, h^{r+s} \cdot m\right]$. To decrypt a ciphertext $C=[W, X, Y]$, compute the plaintext $Y /\left(W^{1 / a} \cdot X^{1 / b}\right)$. Let $p k_{1}=\left(g^{a_{1}}, g^{b_{1}}, g\right)$ and $p k_{2}=\left(h^{a_{2}}, h^{b_{2}}, h\right)$ be two public keys for this cryptosystem.

The basic (naive) re-encryption program from $p k_{1}$ to $p k_{2}$ contains $\left(s k_{1}, p k_{2}\right)$. The program simply decrypts the input using $s k_{1}$ and encrypts the resulting message with $p k_{2}$. Clearly this program exposes both $s k_{1}$ and the underlying plaintext to any third-party executing the re-encryption program.

As a first attempt to obfuscate the basic program, consider the re-encryption program that contains $Z_{1}=a_{2} / a_{1}$ and $Z_{2}=b_{2} / b_{1}$ and re-encrypts the ciphertext $[W, X, Y]$ by computing $\left[W^{Z_{1}}, X^{Z_{2}}, Y\right]$ for $p k_{2}$. (On a different cryptosystem, a similar approach was suggested by Blaze et al. [BBS98.) Unfortunately, this re-encryption program leaks non-black-box information (i.e., does not satisfy the virtual black-box property in Def. 2). For example, the program containing $\left(Z_{1}, Z_{2}\right)$ which translates ciphertexts from Alice to Bob can be transformed into a new program containing $\left(Z_{1}^{-1}, Z_{2}^{-1}\right)$ which translates ciphertexts from Bob to Alice - a feat which black-box access does not allow.

As a second attempt, consider the re-encryption program containing $Z_{1}=$ $h^{a_{2} / a_{1}}$ and $Z_{2}=h^{b_{2} / b_{1}}$. Alice, with $s k_{1}=\left(a_{1}, b_{1}, g\right)$, can compute this program given Bob's public key $p k_{2}=\left(h^{a_{2}}, h^{b_{2}}, h\right)$. (On a different cryptosystem, a similar approach was suggested by Ateniese et al. AFGH06.) The re-encryption program works as follows: on input a ciphertext $[W, X, Y]=\left[g^{a_{1} r}, g^{b_{1} s}, g^{r+s} \cdot m\right]$ under $p k_{1}$, output the ciphertext $\left[\mathbf{e}\left(W, Z_{1}\right), \mathbf{e}\left(X, Z_{2}\right), \mathbf{e}(Y, h)\right]=[E, F, G]$ under $p k_{2}$. To decrypt $[E, F, G]$, the holder of $s k_{2}$ would first compute $Q=$ $G /\left(E^{1 / a_{2}} \cdot F^{1 / b_{2}}\right)$ and then find and output the message $m_{i}$ in the message space $M$ such that $\mathbf{e}\left(m_{i}, h\right)=Q$. Of course, to ensure efficient decryption, this limits the size of the message space $M$ to be a polynomial. Notice the encryption scheme now support two "forms" of ciphertexts - an original form and a 
re-encrypted one, each containing elements from different groups. As a result, a re-encrypted ciphertext cannot be further re-encrypted. The question, though, is whether or not such a program is any closer to being an obfuscation.

To be a secure obfuscation according to our Def. 2, the output of an adversary who is given the obfuscated program must be indistinguishable - even to a distinguisher with oracle access to the re-encryption program-from the output of a simulator given only black-box access to the program. Unfortunately, in the second attempt, knowledge of the public keys $p k_{1}=\left(g^{a_{1}}, g^{b_{1}}, g\right)$ and $p k_{2}=\left(h^{a_{2}}, h^{b_{2}}, h\right)$ easily allows a distinguisher to test whether a program containing $\left(Z_{1}, Z_{2}\right)$ is a valid re-encryption program for these keys by checking that $\mathbf{e}\left(g^{a_{1}}, Z_{1}\right)=\mathbf{e}\left(g, h^{a_{2}}\right)$ and $\mathbf{e}\left(g^{b_{1}}, Z_{2}\right)=\mathbf{e}\left(g, h^{b_{2}}\right)$. We do not know how to construct a simulator that can output a program which also passes this test.

To bypass this problem, we design our re-encryption program to be a probabilistic function of the keys. More specifically, consider the program containing $\left(y^{a_{2} / a_{1}}, y^{b_{2} / b_{1}}, y\right)=\left(Z_{1}, Z_{2}, Z_{3}\right)$ for a randomly selected $y \in \mathbb{G}$. (In the context of point functions, a similar approach was suggested by Canetti [Can97].) Alice can still generate this re-encryption program using only Bob's public key. The re-encryption program becomes: on input $[W, X, Y]=\left[g^{a_{1} r}, g^{b_{1} s}, g^{r+s} \cdot m\right]$ under $p k_{1}$, output the ciphertext under $p k_{2}$ as $\left[\mathbf{e}\left(W, Z_{1}\right), \mathbf{e}\left(X, Z_{2}\right), \mathbf{e}\left(Y, Z_{3}\right), Z_{3}\right]=$ $[E, F, G, H]$. Decryption works as follows: first compute $Q=G /\left(E^{1 / a_{2}} \cdot F^{1 / b_{2}}\right)$ and then output message $m_{i}$ in the message space $M$ such that $\mathbf{e}\left(m_{i}, H\right)=Q$.

This solution has one subtle problem because all ciphertexts produced by the obfuscated re-encryption program include $H=y$ as the fourth component, whereas ciphertexts produced by the decrypt-and-encrypt approach contain a fresh random value in that position. Thus, the obfuscated program does not "preserve the functionality" of the original one. This is easily fixed by having the obfuscated program re-randomize its output by choosing $z \stackrel{r}{\leftarrow} \mathbb{Z}_{q}$ and outputting $\left[E^{z}, F^{z}, G^{z}, H^{z}\right]$. (Note, it is not sufficient that we choose $y$ randomly, since this choice is only made once for all re-encrypted ciphertexts, whereas $z$ is chosen freshly for each re-encryption.)

Even this, however, falls short, because we do not know how to prove this construction is secure. In particular, since the distinguisher has access to a reencryption oracle, it can query the oracle on the values contained in the obfuscated program! Indeed, in the above scheme, there is a specific (complicated) property of valid obfuscated programs that a distinguisher can test for, and we do not know how to construct a simulator that also passes this test.

In order to overcome this final hurdle, our program re-randomizes the input ciphertext before applying the transformation above. If the public key is $\left(g^{a}, g^{b}, g\right)$, and the input ciphertext is $C=[W, X, Y]$, our program re-randomizes $C$ by sampling $r^{\prime}, s^{\prime}$ and computing the ciphertext $\left[W \cdot\left(g^{a}\right)^{r^{\prime}}, X \cdot\left(g^{b}\right)^{s^{\prime}}, Y \cdot g^{r^{\prime}+s^{\prime}}\right]$. Finally, we are able to show this construction meets our obfuscation definition under two reasonable complexity assumptions.

As a final point about our complexity assumptions, because our obfuscation definition only requires average-case obfuscation, we do not have to make the strong complexity assumptions necessary in the constructions of Canetti Can97. 
and Wee Wee05]. Thus, our scheme simultaneously meets a strong theoretical definition while retaining the sensibility associated with standard assumptions and efficient algorithms.

\section{Definitions}

Barak et al. $\mathrm{BGI}^{+} 01$ required that an obfuscator strip programs of non-blackbox information. They formalized this by requiring that any predicate computable from the obfuscated program is also computable from black-box access to it. Goldwasser and Kalai GK05] gave a stronger definition, guaranteeing security in the presence of (dependent) auxiliary input. A formal definition, which we call predicate black-box obfuscation (or predicate obfuscation, for short), follows.

For a family $\mathbf{C}$ of polynomial-size circuits, for a length parameter $n$ let $\mathbf{C}_{n}$ be the circuits in $\mathbf{C}$ with input length $n$ (i.e. $\mathbf{C}=\left\{\mathbf{C}_{n}\right\}$ ).

Definition 1 (Predicate Obfuscation [BGI ${ }^{+}$01, GK05]). An efficient algorithm Obf is a predicate obfuscator for the family $\mathbf{C}=\left\{\mathbf{C}_{n}\right\}$, if it has the following properties:

- Preserving Functionality: There exists a negligible function neg(n), s.t. for all input lengths $n$, for any $C \in \mathbf{C}_{n}$ :

$$
\operatorname{Pr}\left[\exists x \in\{0,1\}^{n}:(\operatorname{Obf}(C))(x) \neq C(x)\right] \leq n e g(n)
$$

The probability is taken over Obf's random coins.

- Polynomial Slowdown: There exists a polynomial $p(n)$ such that for sufficiently large input lengths $n$, for any $C \in \mathbf{C}_{n}$, the obfuscator Obf only enlarges $C$ by a factor of $p:|\operatorname{Obf}(C)| \leq p(|C|)$.

- Predicate Virtual Black-box: For every polynomial sized adversary circuit $\mathcal{A}$, there exists a polynomial size simulator circuit $\operatorname{Sim}$ and a negligible function neg $(n)$, such that for every input length $n$, for every $C \in \mathbf{C}_{n}$, for every predicate $\pi$, for every auxiliary input $z \in\{0,1\}^{q(n)}$ :

$$
\left|\operatorname{Pr}[\mathcal{A}(\operatorname{Obf}(C), z)=\pi(C, z)]-\operatorname{Pr}\left[\operatorname{Sim}^{C}\left(1^{n}, z\right)=\pi(C, z)\right]\right| \leq n e g(n)
$$

The probability is over the coins of the adversary, the simulator and the obfuscator.

As discussed in $₫ 1$, the predicate black-box definition does not guarantee security when obfuscated circuits are used in cryptographic settings. To address this, we introduce the new notion of average-case secure obfuscation:

Definition 2 (Average-Case Secure Obfuscation). An efficient algorithm Obf that takes as input a (probabilistic) circuit and outputs a new (probabilistic) 
circuit, is an average-case secure obfuscator for the family $\mathbf{C}=\left\{\mathbf{C}_{n}\right\}$, if it satisfies the following properties:

- Preserving Functionality: "With overwhelming probability $\operatorname{Obf}(C)$ behaves almost identically to $C$ on all inputs". There exists a negligible function $n e g(n)$, such that for any input length $n$, for any $C \in \mathbf{C}_{n}$ :

$$
\underset{\text { coins of obf }}{\operatorname{Pr}}\left[\exists x \in\{0,1\}^{n}: \Delta((\operatorname{Obf}(C))(x), C(x)) \geq n e g(n)\right] \leq n e g(n)
$$

The distributions $(\operatorname{Obf}(C))(x)$ and $C(x)$ are taken over $\operatorname{Obf}(C)$ 's and $C$ 's random coins respectively. $\Delta$ denotes statistical (L1) distance between distributions.

- Polynomial Slowdown: (identical to Definition 1)

- Average-Case Secure Virtual Black-Box: For any efficient adversary $\mathcal{A}$, there exists an efficient simulator Sim and a negligible function neg $(n)$, such that for every efficient distinguisher D, for every input length $n$ and for every polynomial-size auxiliary input $z$ :

$$
\begin{aligned}
& \left|\begin{array}{l}
\operatorname{Pr}\left[C \stackrel{r}{\leftarrow} \mathbf{C}_{n}: \mathrm{D}^{C}(\mathcal{A}(\operatorname{Obf}(C), z), z)=1\right] \\
\quad-\operatorname{Pr}\left[C \stackrel{r}{\leftarrow} \mathbf{C}_{n}: \mathrm{D}^{C}\left(\operatorname{Sim}^{C}\left(1^{n}, z\right), z\right)=1\right]
\end{array}\right| \leq n e g(n)
\end{aligned}
$$

The probability is over the selection of a random circuit $C$ from $\mathbf{C}_{n}$, and the coins of the distinguisher, the simulator, the oracle and the obfuscator. Note that entities with black-box access to $C$ cannot set $C$ 's random tape.

Note that without loss of generality it is sufficient to require the existence of a simulator for the "dummy" adversary that just outputs its input. This would give an equivalent definition, but it loses some intuitive appeal.

Discussion. Intuitively, Definition 2 guarantees that any attack that a nonblack box adversary can mount using the obfuscated circuit, can also be mounted by a black-box simulator with (oracle) access to the functionality. This new definition differs from the predicate definition in several ways. It considers obfuscation of a random circuit from a family, and furthermore, the circuit families considered can be probabilistic (this allows us to side-step impossibility results, see $\$ 2.2$. We also follow GK05] in requiring that the obfuscation be secure in the presence of (independent) auxiliary input, where the auxiliary input is selected first, and then a random circuit is chosen from the family. Note that the average-case secure virtual black-box requirement of our new definition is incomparable to the predicate black-box requirement of $\left[\mathrm{BGI}^{+} 01\right]$; the latter is weaker in that it only requires that the obfuscator hides predicates, but is stronger in that it provides the predicate distinguisher with the actual program (whereas our definition only gives our predicate distinguisher black-box access).

Finally, we emphasize that in this new definition there are two important sources of randomness. The first source of randomness is in the circuits being obfuscated, which are probabilistic. The second, more subtle, source of randomness is in the selection of a random circuit $C$ from the family $\mathbf{C}_{n}$. The average-case secure virtual black-box requirement guarantees security when a 
circuit is selected from the family by a specific distribution (i.e., the uniform distribution - one should think of this as uniformly choosing random keys for a cryptographic scheme). The predicate black-box definition, on the other hand, guarantees security for every circuit in the family, or (equivalently) for every distribution on circuits. Other work CMR98, DS05] guarantees security for a large class of distributions on circuits from a family, such as every distribution with at least super-logarithmic min-entropy. Our notion of secure obfuscation can be generalized to give security against more general classes of distributions. For clarity, we choose to present the less general definition above.

\subsection{Meaningfulness for Security}

This section serves as an informal discussion of the security guarantee provided by average-case secure obfuscation. As mentioned in $\$ 1$ the definition of obfuscation should be security-preserving in the following sense: "If a cryptographic scheme is secure when the adversary is given black-box access to a program, then it remains secure when the adversary is given the obfuscated program." We claim that for a large class of applications (including re-encryption), average-case secure obfuscation indeed gives this guarantee.

To see this, consider any cryptographic scheme, for which a distinguisher, that has only public information (e.g. public keys) and black-box access to an obfuscated program, can test whether a given adversary can break a scheme (we call this the distinguishable attack property). Many standard cryptographic schemes, such as semantically secure encryption and re-encryption, have this property. For such schemes Definition 2 indeed guarantees that for every adversary that mounts an attack using an obfuscated circuit, there exists a black-box simulator that can mount an attack with a similar success probability. Thus, if the scheme is secure against black-box adversaries, it is also secure against non black-box adversaries that are given the obfuscated program.

To illustrate the meaningfulness of the notion of average-case secure obfuscation, we propose to use the following informal argument as a methodology for constructing secure cryptographic schemes:

If a cryptographic scheme has the following three properties:

1. The scheme is secure against black-box adversaries with oracle access to functionality $X$ selected randomly from a family $F$

2. A distinguisher $D$ with oracle access to $X$ can test whether an adversary $\mathcal{A}$ can break the security guarantee of the scheme (we call this property the distinguishable attack property)

3. There exists an average-case secure obfuscator for a family $C_{F}$ of circuits implementing the functionalities in $F$,

Then the cryptographic scheme is also secure against adversaries who are given an obfuscation of a circuit selected at random from the family $C_{F}$.

As a case study, consider semantically-secure re-encryption (see Def. 3). An attacker is given two relevant public keys and black-box access to a re-encryption 
oracle. The attacker is successful if it can distinguish the encryptions of two different messages (of its choice) under one of the public keys. As with many cryptographic schemes, re-encryption schemes have Property (11). For Property (2), a distinguisher who is given public keys and oracle access to the re-encryption functionality can indeed test whether an adversary has a noticeable chance of mounting a successful attack 1 Thus, for any re-encryption functionality, assuming that Property (3) holds (i.e. there exists an average-case secure obfuscator for some circuit family computing re-encryption), we conclude that the scheme is also secure against adversaries who are given an obfuscated re-encryption circuit. The predicate definition would not let us make such a conclusion.

\subsection{Obfuscating Probabilistic Programs}

In this section we discuss an impossibility result for average-case secure obfuscation of deterministic circuits, and explain how we side-step this impossibility by considering probabilistic circuits. Wee Wee05 observes that the only deterministic circuits that can be obfuscated under strong security-preserving notions of obfuscation are those that are learnable. This result also applies to obfuscating deterministic circuits under Definition 2, To see the intuition behind this result, consider a circuit family $\mathbf{C}$, the "empty" adversary who simply outputs the obfuscated circuit $\operatorname{Obf}(C)$ it gets, and a distinguisher (with black-box access to $C$ ) that outputs 1 only if whatever circuit it gets agrees with $C$ for random inputs 2 Because Obf preserves functionality, the above adversary that outputs $\operatorname{Obf}(C)$ will get the distinguisher to accept with all but negligible probability. To make the distinguisher accept with similar probability, the simulator must learn, from black-box access, a circuit that is (at the very least) very close to $C$ on random inputs. Thus random circuits from $\mathbf{C}$ must be learnable from black-box access. In particular, deterministic circuit families that are not learnable cannot be obfuscated under Definition 2

This impossibility disappears when we consider probabilistic circuit families. This is because the (efficient) distinguisher with black-box access to a probabilistic $C$ and non black-box access to $\operatorname{Obf}(C)$ cannot necessarily distinguish whether the distributions that $C$ and $\operatorname{Obf}(C)$ output on a particular input are statistically close or far. This is similar to the case of encryption (see Goldwasser and Micali GM84]), where only randomness can prevent an adversary from recognizing whether two ciphertexts are encryptions of the same bit. Our obfuscation of re-encryption programs uses this observation. In fact, our simulator outputs a "dummy circuit" that has little to do with the circuit being obfuscated, but is still indistinguishable from the true obfuscated circuit.

\footnotetext{
${ }^{1}$ To do this, the distinguisher simply runs the adversary with the public keys, answering the adversary's re-encryption requests using the re-encryption oracle.

${ }^{2}$ Wee considers different distinguishers that check different inputs.
} 


\section{Algebraic Setting and Assumptions}

Bilinear Groups. Let BMsetup be an algorithm that, on input the security parameter $1^{k}$, outputs the parameters for a bilinear map as $\left(q, g, \mathbb{G}, \mathbb{G}_{T}, \mathbf{e}\right)$, where $\mathbb{G}, \mathbb{G}_{T}$ are groups of prime order $q \in \Theta\left(2^{k}\right)$. The efficient mapping $\mathbf{e}: \mathbb{G} \times \mathbb{G} \rightarrow \mathbb{G}_{T}$ is both bilinear, i.e., for all $g \in \mathbb{G}$ and $a, b \in \mathbb{Z}_{q}, \mathbf{e}\left(g^{a}, g^{b}\right)=\mathbf{e}(g, g)^{a b}$, and non-degenerate, i.e., if $g$ generates $\mathbb{G}$, then $\mathbf{e}(g, g) \neq 1$.

For simplicity, we present our solution using bilinear maps of the form e : $\mathbb{G} \times \mathbb{G} \rightarrow \mathbb{G}_{T}$. Our scheme can also be implemented in the more general setting where e : $\mathbb{G}_{1} \times \mathbb{G}_{2} \rightarrow \mathbb{G}_{T}$ and isomorphisms between $\mathbb{G}_{1}$ and $\mathbb{G}_{2}$ may not be efficiently computable. Galbraith, Paterson, and Smart GPS06 provide more information on various implementation options.

Complexity Assumptions. In this paper, we make the following two complexity assumptions in bilinear groups. When we say two distributions are computationally indistinguishable, we mean with respect to a distinguisher with auxiliary information (which is selected independently of the instance).

Assumption 1 (Strong Diffie Hellman Indistinguishability). Let $\mathbb{G}$ be a group of order $q$ where $q$ is a $k$-bit prime, $g \stackrel{r}{\leftarrow} \mathbb{G}$ and $a, b, c, d \stackrel{r}{\leftarrow} \mathbb{Z}_{q}$. Then the following two distributions are computationally indistinguishable:

$$
\left\{g, g^{a}, g^{b}, g^{c}, g^{a b c}\right\}_{k} \stackrel{c}{\approx}\left\{g, g^{a}, g^{b}, g^{c}, g^{d}\right\}_{k}
$$

This assumption has not been proposed before, but it is implied by the Decision 3-party Diffie-Hellman assumption proposed in BSW06].

Assumption 2 (Decision Linear [BBS04]). Let $\mathbb{G}$ be a group of order $q$ where $q$ is a $k$-bit prime, $f, g, h \stackrel{r}{\leftarrow} \mathbb{G}$ and $a, b, c \stackrel{r}{\leftarrow} \mathbb{Z}_{q}$. Then the following two distributions are computationally indistinguishable:

$$
\left\{f, g, h, f^{a}, g^{b}, h^{a+b}\right\}_{k} \approx\left\{f, g, h, f^{a}, g^{b}, h^{c}\right\}_{k}
$$

\section{A Special Encryption Scheme and Re-encryption Functionality}

In this section, we describe a special encryption scheme and a re-encryption functionality for which we later present a secure obfuscation scheme.

\subsection{A Special Encryption Scheme $\Pi$}

Our special encryption scheme $\Pi$ is described in Fig.1. The encryption algorithm supports two forms of ciphertexts and takes an additional input $\beta \in\{0,1\}$ to choose between them. For the first form, encryption and decryption work as per the Boneh et al. BBS04 construction. For the second form, the encryption and decryption are novel and relevant for re-encryption. Note that this encryption system also requires the message space $M$ to be a subset of $\mathbb{G}$ which is of size polynomial in $k$. The semantic security of this scheme will be proven in Thm. 3 . 
Common: For a security parameter $1^{k}$, let $\left(q, g, \mathbb{G}, \mathbb{G}_{T}, \mathbf{e}\right) \leftarrow \operatorname{BMsetup}\left(1^{k}\right)$ be a common parameter and let $M \subset \mathbb{G}$ where $|M|=O($ poly $(k))$ be the message space.

$\operatorname{KeyGen}\left(1^{k},\left(q, g, \mathbb{G}, \mathbb{G}_{T}, \mathbf{e}\right)\right)$ :

1. Randomly select a new generator $h \stackrel{r}{\leftarrow} \mathbb{G}$ and random $a, b \stackrel{r}{\leftarrow} \mathbb{Z}_{q}$.

2. Output $p k=\left(h^{a}, h^{b}, h\right)$ and $s k=(a, b, h)$.

$\operatorname{Enc}(p k, \beta, m)$ :

1. Parse $p k=\left(h^{a}, h^{b}, h\right)$.

2. Choose random $r, s \stackrel{r}{\leftarrow} \mathbb{Z}_{q}$.

3. If $\beta=0$, output the ciphertext $\left[0,\left(h^{a}\right)^{r},\left(h^{b}\right)^{s}, h^{r+s} \cdot m, 0\right]$.

4. If $\beta=1$, then choose a random $t \stackrel{r}{\leftarrow} \mathbb{G}$, and output the ciphertext $\left[1, \mathbf{e}\left(\left(h^{a}\right)^{r}, t\right), \mathbf{e}\left(\left(h^{b}\right)^{s}, t\right), \mathbf{e}\left(h^{r+s} \cdot m, t\right), t\right]$.

$\operatorname{Dec}(s k,[s, W, X, Y, Z])$ :

1. Parse $s k=(a, b, h)$.

2. If $s=0$, then output $Y /\left(W^{1 / a} \cdot X^{1 / b}\right)$.

3 . If $s=1$, then

(a) Compute $Q=Y /\left(W^{1 / a} \cdot X^{1 / b}\right)$.

(b) For each $m \in M$, test if $\mathbf{e}(m, Z)=Q$. If so, output $m$ and halt.

Fig. 1. Encryption Scheme $\Pi$

\subsection{Re-encryption Functionality}

Recall that obfuscation is with respect to a class of circuits. We now define a special class of re-encryption circuits for the encryption scheme $\Pi$ which can be easily analyzed.

Let $\left(p k_{1}, s k_{1}\right)$ and $\left(p k_{2}, s k_{2}\right)$ be two keys pairs which were generated by running KeyGen on independent random tapes. When given an honestly-generated ciphertext encrypted under $p k_{1}$, a re-encryption circuit decrypts the ciphertext and then re-encrypts the resulting message under a second public key $p k_{2}$. For technical reasons, we also require the circuit to produce the pairs of public keys for which it transforms ciphertexts.

More formally, the re-encryption circuit $F_{s k_{1}, p k_{2}}$, when run on input $c_{1}=$ $\operatorname{Enc}\left(p k_{1}, 0, m\right)$ for any message $m \in M$, computes $m \leftarrow \operatorname{Dec}\left(s k_{1}, c_{1}\right)$, then computes $c_{2} \leftarrow \operatorname{Enc}\left(p k_{2}, 1, m\right)$, and finally outputs $c_{2}$. When $F_{s k_{1}, p k_{2}}$ is run on input the special symbol keys, it outputs the ordered pair of public keys $\left(p k_{1}, p k_{2}\right)$. For ciphertexts corresponding to messages in not in $M$, the circuit returns a randomized ciphertext of the second form for the same message. For other ill-formed inputs, it returns $\perp$.

Furthermore, let $C_{s k_{1}, p k_{2}}$ be the same as $F_{s k_{1}, p k_{2}}$ with the exception that the values $s k_{1}$ and $p k_{2}$ can be read from the circuit description. This property is easy to achieve by adding a "message" section to the circuit which does not affect the circuit's output, but encodes a message, with say, AND gates encoding a 1 and or gates encoding a 0 . We now define the family of circuits:

$$
C_{k}=\left\{C_{s k_{1}, p k_{2}} \mid\left(p k_{1}, s k_{1}\right) \leftarrow \operatorname{KeyGen}\left(1^{k}\right),\left(p k_{2}, s k_{2}\right) \leftarrow \operatorname{KeyGen}\left(1^{k}\right)\right\}
$$




\subsection{Security for Re-encryption}

We generalize the standard notion of indistinguishability [GM84 for encryption schemes by allowing the adversary to have access to a re-encryption oracle. In particular, the following definition captures the notion that "given a ciphertext $y$ and black-box access to a re-encryption circuit, an adversary does not learn any information about the plaintext corresponding to $y$."

Definition 3 (IND-security with Oracle $C_{s k_{1}, p k_{2}}$ ). Let $\Pi$ be an encryption scheme and let the random variable $\operatorname{IND}_{b}(\Pi, \mathcal{A}, k)$ where $b \in\{0,1\}, \mathcal{A}=$ $\left(\mathcal{A}_{1}, \mathcal{A}_{2}\right)$ and $k \in \mathbb{N}$ denote the result of the following probabilistic experiment:

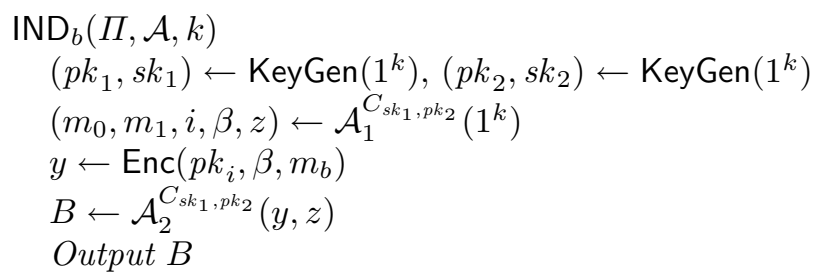

Scheme $\Pi$ is indistinguishable under a chosen-plaintext attack if $\forall$ p.p.t. algorithms $\mathcal{A}$ the following two ensembles are computationally indistinguishable:

$$
\left\{\operatorname{IND}_{0}(\Pi, \mathcal{A}, k)\right\}_{k} \stackrel{c}{\approx}\left\{\operatorname{IND}_{1}(\Pi, \mathcal{A}, k)\right\}_{k}
$$

Remark 1. For simplicity, we allow the adversary to pick the key $p k_{i}$ under which the challenge is encrypted and the form $\beta$ of the encryption. By a standard hybrid argument, the above definition is equivalent to one in which the adversary is given four encryptions of the challenge message - one per key and per form.

Theorem 3. The encryption scheme $\Pi$ (in Fig. 1) is an indistinguishablesecure encryption scheme with respect to oracle $C_{s k_{1}, p k_{2}}$ under the Decision Linear assumption in $\mathbb{G}$.

The proof sketch is given in Appendix $\mathrm{A}$

\section{The Obfuscator for Re-encryption}

In Fig. 2, we describe an obfuscator Obf for the class of re-encryption circuits $C_{k}$ relative to the encryption scheme $\Pi$ defined in the previous section.

\subsection{Main Result}

Theorem 4. The obfuscator in Fig. 2 is a secure obfuscator for family $C_{k}$.

Proof sketch. Let $p k_{1}=\left(g^{a_{1}}, g^{b_{1}}, g\right)$ and $p k_{2}=\left(h^{a_{2}}, h^{b_{2}}, h\right)$ with appropriately defined secret keys. Let $C$ denote the re-encryption circuit $C_{s k_{1}, p k_{2}}$, and let $R \leftarrow \operatorname{Obf}(C)$ be an obfuscated version of $C$. 
Algorithm Obf, on input a circuit $C_{s k_{1}, p k_{2}} \in C_{k}$,

1. Reads $s k_{1}=\left(a_{1}, b_{1}, g\right)$ and $p k_{2}=\left(h^{a_{2}}, h^{b_{2}}, h\right)$ from the description of $C_{s k_{1}, p k_{2}}$.

2. Selects a random integer $z \stackrel{r}{\leftarrow} \mathbb{Z}_{q}^{*}$ and compute the re-encryption tuple $\left(Z_{1}, Z_{2}, Z_{3}\right)=\left(\left(h^{a_{2}}\right)^{z / a_{1}},\left(h^{b_{2}}\right)^{z / b_{1}}, h^{z}\right)$.

3. Constructs and outputs an obfuscated circuit $R_{s k_{1}, p k_{2}}$ that contains the values $p k_{1}, p k_{2}, Z_{1}, Z_{2}, Z_{3}$ and does the following:

- On input keys, output $p k_{1}=\left(g^{a_{1}}, g^{b_{1}}, g\right)$ and $p k_{2}=\left(h^{a_{2}}, h^{b_{2}}, h\right)$.

- On input a 5 -tuple $[0, W, X, Y, 0]$ where $W, X, Y \in \mathbb{G}$, then:

(a) Select input re-randomization values $r, s \stackrel{r}{\leftarrow} \mathbb{Z}_{q}^{*}$.

(b) Re-randomize the input as $W^{\prime} \leftarrow W \cdot\left(g^{a_{1}}\right)^{r}, X^{\prime} \leftarrow X \cdot\left(g^{b_{1}}\right)^{s}$, and $Y^{\prime} \leftarrow Y \cdot g^{r+s}$.

(c) Compute $E \leftarrow \mathbf{e}\left(W^{\prime}, Z_{1}\right)$.

(d) Compute $F \leftarrow \mathbf{e}\left(X^{\prime}, Z_{2}\right)$.

(e) Compute $G \leftarrow \mathbf{e}\left(Y^{\prime}, Z_{3}\right)$.

(f) Select an output re-randomization value $y \stackrel{r}{\leftarrow} \mathbb{Z}_{q}^{*}$.

(g) Output the ciphertext $\left[1, E^{y}, F^{y}, G^{y}, Z_{3}^{y}\right]$.

- Otherwise return $\perp$.

Fig. 2. Obfuscator Obf for Re-encryption circuits for $\Pi$

Preserving Functionality. Consider any $C \in C_{k}$ and let circuit $R \leftarrow \operatorname{Obf}(C)$. We claim that the output distributions of $C$ and $R$ are statistically close (in fact, identical). To see this, we must consider three classes of inputs. First, for any message $m \in M$, observe that

$$
\operatorname{Enc}\left(p k_{1}, 0, m\right)=\left[0, g^{a_{1} r}, g^{b_{1} s}, g^{r+s} \cdot m, 0\right]
$$

for a randomly chosen $r, s \stackrel{r}{\leftarrow} \mathbb{Z}_{q}^{*}$. When such a ciphertext is fed as input to $R$, the circuit outputs

$$
\left[1, \mathbf{e}\left(g^{a_{1}\left(r+r^{\prime}\right)}, h^{a_{2} z / a_{1}}\right)^{y}, \mathbf{e}\left(g^{b_{1}\left(s+s^{\prime}\right)}, h^{b_{2} z / b_{1}}\right)^{y}, \mathbf{e}\left(g^{r+s+r^{\prime}+s^{\prime}} \cdot m, h^{z}\right)^{y}, h^{z y}\right]
$$

for randomly chosen $r^{\prime}, s^{\prime}, y \stackrel{r}{\leftarrow} \mathbb{Z}_{q}^{*}$. Substituting $\bar{r}=\frac{r+r^{\prime}}{\ell}, \bar{s}=\frac{s+s^{\prime}}{\ell}, \bar{t}=h^{y z}$, and $\ell$ is such that ${ }^{3} g^{\ell}=h$, this 5 -tuple can be re-written as

$$
\left[1, \mathbf{e}\left(h^{a_{2} \bar{r}}, \bar{t}\right), \mathbf{e}\left(h^{b_{2} \bar{r}}, \bar{t}\right), \mathbf{e}\left(h^{\bar{r}+\bar{s}} \cdot m, \bar{t}\right), \bar{t}\right]
$$

which is identically distributed to the output of $\operatorname{Enc}\left(p k_{2}, 1, m\right)$. Second, the same holds for all $m \in \mathbb{G} \backslash M$. Lastly, for keys and junk input, the outputs are identical.

Polynomial slowdown. This property follows by inspection because the obfuscated circuit computes a few bilinear maps and exponentiations.

${ }^{3}$ We do not need to compute $\ell$ explicitly. 
Virtual Blackbox. In order to satisfy the virtual black-box property, it suffices to only consider the "dummy" adversary. Thus, we must construct a simulator $\operatorname{Sim}^{C}\left(1^{k}, z\right)$ such that for all distinguishers $D^{C}$ which take as input an obfuscated circuit $R$ and auxiliary input $z$,

$$
\left|\operatorname{Pr}\left[D^{C}(\operatorname{Obf}(C), z)=1\right]-\operatorname{Pr}\left[D^{C}\left(\operatorname{Sim}^{C}\left(1^{k}, z\right), z\right)=1\right]\right|<n e g(k) .
$$

Let us define the simulator $\operatorname{Sim}^{C}\left(1^{k}, z\right)$ as follows:

1. Query the oracle $C$ on keys to get $p k_{1}, p k_{2}$.

2. Sample $Z_{1}^{\prime}, Z_{2}^{\prime}, Z_{3}^{\prime} \stackrel{r}{\leftarrow} \mathbb{G}$.

3. As in Step (3) of the Obf algorithm, create and output a circuit $R^{\prime}$ using the values $\left(p k_{1}, p k_{2}, Z_{1}^{\prime}, Z_{2}^{\prime}, Z_{3}^{\prime}\right)$.

Notice that $\operatorname{Sim}^{C}$ produces a circuit which does not correctly compute the reencryption function. However, we now show that under appropriate complexity assumptions, no p.p.t. distinguisher $D^{C}$ will notice.

Towards this goal, notice that the output of $D^{C}(\operatorname{Obf}(C), z)$ is distributed identically to $\operatorname{Nice}\left(D^{C}, k, z\right)$ and the output of $D^{C}\left(\operatorname{Sim}^{C}\left(1^{k}, z\right)\right)$ is distributed identically to $\operatorname{Junk}\left(D^{C}, k, z\right)$ where

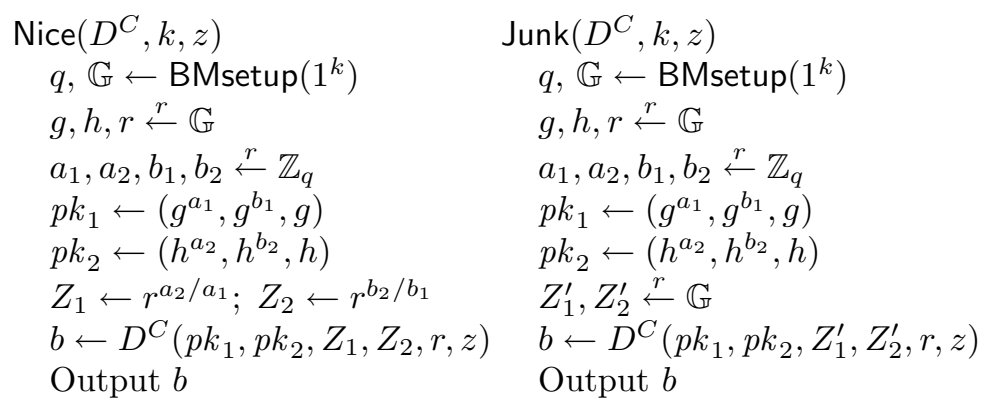

In the above experiments, the oracle $C$ represents the re-encryption oracle for the public keys $p k_{1}$ to $p k_{2}$ which are chosen in the experiment. There is a slight abuse of notation here; when we write $\operatorname{expt}\left(D^{C}, k, z\right)$ we mean that the distinguisher $D$ has oracle access to $C_{s k_{1}, p k_{2}}$ for the keys $s k_{1}, p k_{2}$ chosen in the experiment. The virtual blackbox property follows immediately from the following lemma.

Lemma 1. Under the SDHI and Decision Linear assumptions, for all p.p.t. distinguishers $D$ and auxiliary information $z$, the following two distributions are statistically close.

$$
\left\{\operatorname{Nice}\left(D^{C}, k, z\right)\right\}_{k} \quad \text { and } \quad\left\{\operatorname{Junk}\left(D^{C}, k, z\right)\right\}_{k}
$$

Proof Outline. We prove this lemma in a series of incremental steps. We begin with a simple indistinguishability problem and incrementally add elements and provide access to various oracles until the experiments are equivalent to their final form in Lemma 1. Let us now start with a claim which relates the SDHI problem 
to a simple indistinguishability problem: (In all of the following experiments, we implicitly generate $q, \mathbb{G} \leftarrow \operatorname{BMsetup}\left(1^{k}\right)$ and each experiment is indexed by $k$ and $z$ although we omit this extra notation when the context is clear.)

Proposition 1. Under the SDHI assumption, Nice $k_{k, z}^{(1)} \approx$ Junk $_{k, z}^{(1)}$ where

Nice ${ }^{(1)}$ : Proceeds as Nice except that the output is $\left(g^{a_{1}}, g, h^{a_{2}}, h, Z_{1}, r, z\right)$.

Junk $^{(1)}$ : Proceeds as Junk except that the output is $\left(g^{a_{1}}, g, h^{a_{2}}, h, Z_{1}^{\prime}, r, z\right)$.

If there exists a distinguisher $D$ which distinguishes Nice ${ }^{(1)}$ from Junk ${ }^{(1)}$ with advantage $\varepsilon$, then there exists an distinguisher $D^{\prime}$ which solves the SDHI problem with the same advantage (in roughly the same time).

Proof sketch. The algorithm $D^{\prime}\left(g, g^{a}, g^{b}, g^{c}, Q, z\right)$ works as follows:

1. $D^{\prime}$ chooses a random $w \stackrel{r}{\leftarrow} \mathbb{Z}_{q}$.

2. $D^{\prime}$ runs $D\left(g^{w},\left(g^{b}\right)^{w}, g^{a}, g, Q, g^{c}, z\right)$ and echoes the response.

Consider $a_{1}=1 / b, a_{2}=a$ and $r=g^{c}$. Thus, if $Q=g^{a b c}$, then we have $Q=r^{a b}=r^{a_{2} / a_{1}}$ in which case the input to $D$ is identically distributed to Nice ${ }^{(1)}$. Otherwise, $Q$ is equal to $r^{t}$ for some random $t$ and the input to $D$ is identically distributed to Junk ${ }^{(1)}$.

We now extend Proposition 1 to include more input values.

Proposition 2. Under the SDHI assumption, Nice ${ }_{k, z}^{(2)} \approx$ Junk $_{k, z}^{(2)}$ where

Nice $^{(2)}$ : Same as Nice except that the output is $\left(p k_{1}, p k_{2}, Z_{1}, Z_{2}, r, z\right)$.

Junk $^{(2)}$ : Same as Junk except that the output is $\left(p k_{1}, p k_{2}, Z_{1}^{\prime}, Z_{2}^{\prime}, r, z\right)$.

Proof sketch. Consider the hybrid distribution $T^{(2)}$ which is the same as Nice ${ }^{(2)}$ except that $Z_{2}^{\prime} \stackrel{r}{\leftarrow} \mathbb{G}$ and the output is $\left(p k_{1}, p k_{2}, Z_{1}, Z_{2}^{\prime}, r, z\right)$. If $\mathrm{Nice}^{(2)}$ and Junk $^{(2)}$ are distinguishable with advantage $\varepsilon$, then either Nice ${ }^{(2)}$ and $T^{(2)}$ or $T^{(2)}$ and Junk ${ }^{(2)}$ are distinguishable by algorithm $D$ with advantage $\varepsilon / 2$. Either case implies a distinguisher for Nice ${ }^{(1)}$ from Junk ${ }^{(1)}$. In the later case, this involves picking $b_{1}, b_{2} \in \mathbb{Z}_{q}$ to form public keys, picking $Z_{2}^{\prime}$ randomly, and using the input instance from Nice ${ }^{(1)}$ (or Junk ${ }^{(1)}$ ) to simulate the input distribution for $D$. The former case does the same, but swaps the role of $a_{i}$ and $b_{i}$.

Towards the proof of our main theorem, we now extend Prop. 2 by providing the distinguisher with an oracle which returns a five-tuple of random values which works as follows. On input $[0, W, X, Y, 0]$, where $W, X, Y \in \mathbb{G}, \mathcal{R}$ selects three random values $E, F, G \stackrel{r}{\leftarrow} \mathbb{G}_{T}$ and a random value $H \stackrel{r}{\leftarrow} \mathbb{G}$ and returns $[1, E, F, G, H]$. Otherwise, $\mathcal{R}$ returns $\perp$. Intuitively, oracle $\mathcal{R}$ outputs only random values and thus should not help any distinguisher.

Proposition 3. Under the SDHI assumption, Nice $_{k, z}^{(3)} \stackrel{s}{\approx} \mathrm{Junk}_{k, z}^{(3)}$ where

Nice ${ }^{(3)}$ : Same as $\operatorname{Nice}\left(D^{\mathcal{R}}, k, z\right)$.

Junk $^{(3)}$ : Same as Junk $\left(D^{\mathcal{R}}, k, z\right)$.

(That is, the distinguishers have oracle access to $\mathcal{R}$ instead of $C$ and unlike the ${ }^{(2)}$-experiments which output a tuple, these experiments output a bit.) 
Proof sketch. The oracle $\mathcal{R}$ can be perfectly simulated without any auxiliary information. Thus, for any $D^{R}$, there exists another non-oracle distinguisher $D^{\prime}$ (which internally runs $D$ while perfectly simulating $\mathcal{R}$ to $D$ ) whose output distribution is identical to $D$. Proposition 2 therefore implies that for all distinguishers $D^{R}$, Nice ${ }^{(2)} \stackrel{c}{\approx}$ Junk $^{(2)}$ which implies Nice ${ }^{(3)} \stackrel{s}{\approx}$ Junk $^{(3)}$ (since the later experiment outputs a bit)

We now return to the first experiments in which the distinguisher has oracle access to the re-encryption circuit $C$.

Proposition 4. For any p.p.t. distinguisher D, let

$$
\begin{aligned}
& \alpha(k, z)=\operatorname{Adv}\left(\operatorname{Nice}\left(D^{C}, k, z\right), \operatorname{Junk}\left(D^{C}, k, z\right)\right) \\
& \beta(k, z)=\operatorname{Adv}\left(\operatorname{Nice}\left(D^{\mathcal{R}}, k, z\right), \operatorname{Junk}\left(D^{\mathcal{R}}, k, z\right)\right)
\end{aligned}
$$

be the advantage 4 that $D$ has in distinguishing Nice from Junk given either a re-encrypting oracle $C$ or a random oracle $\mathcal{R}$ respectively. There exists a p.p.t. algorithm $\mathcal{A}$ which decides the Decision Linear problem with probability at least $\frac{1}{2}+\frac{1}{4}(\alpha(k, z)-\beta(k, z))$.

Proof. Without loss of generality, assume that $\alpha>\beta$. (If not, then we flip the way $\mathcal{A}$ guesses in its final step.) The algorithm $\mathcal{A}$ takes as input, a Decision Linear instance $\Gamma=\left(h_{1}, h_{2}, h, h_{1}^{x}, h_{2}^{y}, Q\right)$ and auxiliary information $z$, and:

1. $\mathcal{A}$ samples a challenge bit $c \stackrel{r}{\leftarrow}\{0,1\}$ to pick whether to run Nice or Junk.

2. $\mathcal{A}$ samples integers $a, b, u \stackrel{r}{\leftarrow} \mathbb{Z}_{q}$ and group elements $g, Z_{1}^{\prime}, Z_{2}^{\prime}, Z_{3}^{\prime} \stackrel{r}{\leftarrow} \mathbb{G}$.

3. $\mathcal{A}$ sets $p k_{1}=\left(g^{a}, g^{b}, g\right)$ and $p k_{2}=\left(h_{1}, h_{2}, h\right)$ and computes a valid reencryption tuple $\left(Z_{1}, Z_{2}, Z_{3}\right)$ by $Z_{1} \leftarrow h_{1}^{u / a}, Z_{2} \leftarrow h_{2}^{u / b}$, and $Z_{3} \leftarrow h^{u}$.

4. If $c=1$, then $\mathcal{A}$ runs $D^{\mathcal{O}}\left(p k_{1}, p k_{2}, Z_{1}, Z_{2}, Z_{3}, z\right)$ where $\mathcal{O}$ is defined below. If $c=0$, then $\mathcal{A}$ runs $D^{\mathcal{O}}\left(p k_{1}, p k_{2}, Z_{1}^{\prime}, Z_{2}^{\prime}, Z_{3}^{\prime}, z\right)$.

When $D$ queries the oracle $\mathcal{O}$ on input $[0, W, X, Y, 0], \mathcal{A}$ responds as follows:

(a) Sample input re-randomization values $r, s, t \stackrel{r}{\leftarrow} \mathbb{Z}_{q}$.

(b) Re-randomize the input as $W^{\prime} \leftarrow W \cdot g^{a r}, X^{\prime} \leftarrow X \cdot g^{b s}$, and $Y^{\prime} \leftarrow Y \cdot g^{r+s}$.

(c) Compute $E \leftarrow \mathbf{e}\left(W^{\prime}, Z_{1}\right) \cdot \mathbf{e}\left(g, h_{1}^{t x}\right)$.

(d) Compute $F \leftarrow \mathbf{e}\left(X^{\prime}, Z_{2}\right) \cdot \mathbf{e}\left(g, h_{2}^{t y}\right)$.

(e) Compute $G \leftarrow \mathbf{e}\left(Y^{\prime}, Z_{3}\right) \cdot \mathbf{e}\left(g, Q^{t}\right)$.

(f) Sample output re-randomization value $\ell \stackrel{r}{\leftarrow} \mathbb{Z}_{q}$.

(g) Respond with the ciphertext $\left[1, E^{\ell}, F^{\ell}, G^{\ell}, Z_{3}^{\ell}\right]$.

Whenever $D$ queries its oracle on input keys, $\mathcal{A}$ responds with $p k_{1}$ and $p k_{2}$, and on all other queries, $\mathcal{A}$ responds with $\perp$.

5. Eventually $D$ outputs $c^{\prime} \in\{0,1\}$. If $c=c^{\prime}, \mathcal{A}$ outputs 1 (i.e., it guesses that $\left.Q=h^{x+y}\right)$. Else if $c \neq c^{\prime}$, then $\mathcal{A}$ outputs 0 (i.e., it guesses that $Q \neq h^{x+y}$ ).

${ }^{4}$ By advantage, we mean the following. Suppose $D_{0}, D_{1}$ are two probability distributions. Then for any adversary $\mathcal{A}$, the advantage in distinguishing $D_{0}$ from $D_{1}$ is defined as: $\operatorname{Adv}_{\mathcal{A}}\left(D_{0}, D_{1}\right)=\left|\operatorname{Pr}\left[x_{0} \stackrel{r}{\leftarrow} D_{0}: \mathcal{A}\left(x_{0}\right)=1\right]-\operatorname{Pr}\left[x_{1} \stackrel{r}{\leftarrow} D_{1}: \mathcal{A}\left(x_{1}\right)=1\right]\right|$. 
Note that $\mathcal{A}$ almost mimics the real obfuscated program. The difference is that when computing (4c)-(4e), additional terms are multiplied in to the ciphertext. When the $\Gamma$ instance is a decision linear tuple, then these operations simply contribute to additional re-randomization of the ciphertext (this does not change the ciphertext distribution). However, if $\Gamma$ is not a decision linear instance, then these operations make $E, F, G$ a random 3-tuple that is also independent of $Z_{3}$. This proof step is essential.

Claim: If $\Gamma$ is a decision linear instance, then $\operatorname{Pr}[\mathcal{A}(\Gamma)=1]=\frac{1}{2}+\alpha(k, z) / 2$. Proof of Claim: When $Q=h^{x+y}$, then $\mathcal{A}$ perfectly simulates Nice ${ }^{C}$ or Junk ${ }^{C}$ towards the algorithm $D$. The key point is to recognize that $\left(h_{1}, h_{2}, h\right)$ can be interpreted as a randomly generated public key since $h_{1}, h_{2}$ can be rewritten as $h_{1}=h^{e_{1}}$ and $h_{2}=h^{e_{2}}$ for some (unknown) $e_{1}, e_{2}$. Since the re-encryption tuple $Z_{1}, Z_{2}, Z_{3}$ is also a valid re-encryption tuple for $p k_{1} \rightarrow p k_{2}$, the input parameters to $D$ in step 4 are identically distributed to the inputs to $D$ in either experiment Nice or Junk. Moreover, the response to an oracle query on keys is also identically distributed. All that remains is to show that the responses $\mathcal{A}$ provides to oracle queries on $[0, W, X, Y, 0]$ are also identically distributed. This last point follows by inspection because $Q=h^{x+y}$ and $Z_{1}, Z_{2}, Z_{3}$ are a valid re-encryption tuple. A simple probability analysis completes the result:

$$
\begin{aligned}
\operatorname{Pr}[\mathcal{A}(\Gamma)=1 \mid \Gamma \in D L] & =\frac{1}{2}\left(\operatorname{Pr}\left[\operatorname{Nice}\left(D^{C}\right)=1\right]+\operatorname{Pr}\left[\operatorname{Junk}\left(D^{C}\right)=0\right]\right) \\
& =\frac{1}{2}\left(\operatorname{Pr}\left[\operatorname{Nice}\left(D^{C}\right)=1\right]+1-\operatorname{Pr}\left[\operatorname{Junk}\left(D^{C}\right)=1\right]\right) \\
& =\frac{1}{2}+\frac{\operatorname{Adv}\left(\operatorname{Nice}\left(D^{C}\right), \operatorname{Junk}\left(D^{C}\right)\right)}{2} \\
& =\frac{1}{2}+\frac{\alpha}{2}
\end{aligned}
$$

Claim: If $\Gamma$ is not a decision linear instance, then $\operatorname{Pr}[\mathcal{A}(\Gamma)=1]=\frac{1}{2}+\beta(k, z) / 2$. Proof of Claim: This proof is almost identical to the previous one. The only difference is we must show that responses to the oracle queries return four randomly selected group elements. Let us denote by $\omega, \chi, \gamma, v$ the values such that $W=g^{\omega}, X=g^{\chi}, Y=g^{\gamma}$ and $Q=h^{v}$, and by $e_{1}, e_{2}$ the values such that $h_{1}=h^{e_{1}}$ and $h_{2}=h^{e_{2}}$. Observe that the elements returned by the oracle are

$$
\begin{aligned}
& E=\left[\mathbf{e}\left(W \cdot g^{a r}, h_{1}^{u / a}\right) \cdot \mathbf{e}\left(g, h_{1}^{t x}\right)\right]^{\ell}=\mathbf{e}(g, h)^{\ell e_{1}[\omega u / a+t x]+r z \ell e_{1}} \\
& F=\left[\mathbf{e}\left(X \cdot g^{b s}, h_{2}^{u / b}\right) \cdot \mathbf{e}\left(g, h_{2}^{t y}\right)\right]^{\ell}=\mathbf{e}(g, h)^{\ell e_{2}[\chi u / b+t y]+s z \ell e_{2}} \\
& G=\left[\mathbf{e}\left(Y \cdot g^{r+s}, h^{u}\right) \cdot \mathbf{e}\left(g, Q^{t}\right)\right]^{\ell}=\mathbf{e}(g, h)^{\ell[(\gamma+r+s) u]+t v \ell} \\
& H=h^{u \ell}
\end{aligned}
$$

Since $r, s, t, \ell$ are fresh independently selected values, then $E, F, G, H$ will also be independent on every invocation of the oracle. 
Proof of Lemma 1, By the decision linear assumption and Prop. 4, it follows that $|\alpha(k, z)-\beta(k, z)|$ is negligible. By Prop. 3. $\beta(k, z)$ must be a negligible function, and therefore, so too must $\alpha(k, z)$. This establishes the lemma.

\section{Acknowledgments}

We would like to thank Ran Canetti for suggesting the problem and the TCC 2007 anonymous reviewers for their helpful comments.

\section{References}

[AFGH06] Giuseppe Ateniese, Kevin Fu, Matthew Green, and Susan Hohenberger. Improved Proxy Re-encryption Schemes with Applications to Secure Distributed Storage. ACM Trans. on Information and System Security, 9(1):1-30, February 2006. Previously, in NDSS, pages 29-43, 2005.

[AW05] Ben Adida and Douglas Wikström. How to shuffle in public. Cryptology ePrint Archive, Report 2005/394, 2005. http://eprint.iacr.org/

[BBS98] Matt Blaze, Gerrit Bleumer, and Martin Strauss. Divertible protocols and atomic proxy cryptography. In EUROCRYPT '98, volume 1403 of LNCS, pages 127-144, 1998.

[BBS04] Dan Boneh, Xavier Boyen, and Hovav Shacham. Short Group Signatures Using Strong Diffie Hellman. In CRYPTO, volume 3152, pages 41-55, 2004.

[BGI $\left.{ }^{+} 01\right]$ Boaz Barak, Oded Goldreich, Russell Impagliazzo, Steven Rudich, Amit Sahai, Salil P. Vadhan, and Ke Yang. On the (im)possibility of obfuscating programs. In CRYPTO '01, volume 2139 of LNCS, pages 1-18, 2001.

[BS97] Matt Blaze and Martin Strauss. Atomic proxy cryptography. Technical report, AT\&T Research, 1997.

[BSW06] Dan Boneh, Amit Sahai, and Brent Waters. Fully collusion resistant traitor tracing with short ciphertexts and private keys. In EUROCRYPT '06, volume 4004 of LNCS, pages 573-592, 2006.

[Can97] Ran Canetti. Towards realizing random oracles: Hash functions that hide all partial information. In CRYPTO, volume 1294, pages 455-469, 1997.

[CMR98] Ran Canetti, Daniele Micciancio, and Omer Reingold. Perfectly one-way probabilistic hash functions (preliminary version). In STOC, pages 131140, 1998.

[DI03] Yevgeniy Dodis and Anca Ivan. Proxy cryptography revisited. In NDSS, 2003.

[DS05] Yevgeniy Dodis and Adam Smith. Correcting errors without leaking partial information. In STOC '05, pages 654-663, 2005.

[GK05] Shafi Goldwasser and Yael Tauman Kalai. On the impossibility of obfuscation with auxiliary input. In FOCS '05, pages 553-562, 2005.

[GM84] Shafi Goldwasser and Silvio Micali. Probabilistic encryption. Journal of Computer and System Sciences, 28(2):270-299, 1984. Previously, in STOC, pages 365-377, 1982.

[GPS06] Steven D. Galbraith, Kenneth G. Paterson, and Nigel P. Smart. Pairings for cryptographers, 2006. Cryptology ePrint Archive: Report 2006/165.

[Had00] Satoshi Hada. Zero-knowledge and code obfuscation. In ASIACRYPT '00, volume 1976 of LNCS, pages 443-457, 2000. 
[MO97] Masahiro Mambo and Eiji Okamoto. Proxy Cryptosystems: Delegation of the Power to Decrypt Ciphertexts. IEICE Trans. Fund. Electronics Communications and Computer Science, E80-A/1:54-63, 1997.

[OS05] Rafail Ostrovsky and William E. Skeith III. Private searching on streaming data. In CRYPTO, volume 3621 of LNCS, pages 223-240, 2005.

[Pas06] Rafael Pass, 2006. Personal Communication.

[Smi05] Tony Smith. DVD Jon: buy DRM-less Tracks from Apple iTunes, March 18, 2005. http://www.theregister.co.uk/2005/03/18/itunes_pymusique.

[Wee05] Hoeteck Wee. On obfuscating point functions. In STOC, pages 523-532, 2005.

\section{A Proof of Security for Encryption Scheme}

Proof sketch.[of Thm. 3] Let us first argue that $\Pi$ is an encryption scheme, i.e., it is perfectly complete. When $\beta=0$, this follows from the BBS scheme. For the second form of ciphertexts, on input $[1, E, F, G, H]$, the decryption algorithm first computes $Q=\frac{G}{E^{1 / a_{2}} \cdot F^{1 / b_{2}}}$, which by inspection is equal to $\mathbf{e}(m, H)$. The decryption algorithm loops over each (of the polynomially many) $m_{i} \in M$ and tests whether $\mathbf{e}\left(m_{i}, H\right)=Q$ and therefore eventually recovers $m$ as required.

To argue that the scheme meets the security definition, suppose adversary $\mathcal{A}=\left(\mathcal{A}_{1}, \mathcal{A}_{2}\right)$ and distinguisher $D$ has advantage $\varepsilon$ in distinguishing $\operatorname{IND}_{0}(\cdots)$ from $\operatorname{IND}_{1}(\cdots)$. Then, we construct an adversary $\mathcal{A}^{\prime}$ that decides the Decision Linear problem with advantage $\varepsilon / 4$ as follows. Let $\Gamma=\left(h_{1}, h_{2}, h, h_{1}^{x}, h_{2}^{y}, Q\right)$ be a DL instance; $\mathcal{A}^{\prime}$ works as follows:

1. Sample $a, b, c \stackrel{r}{\longleftarrow} \mathbb{Z}_{q}$.

2. Set $p k_{1}=\left(h_{1}, h_{2}, h\right)$ and $p k_{2}=\left(h_{1}^{a c}, h_{2}^{b c}, h^{c}\right)$.

3. $\operatorname{Run} \mathcal{A}_{1}^{\mathcal{O}}\left(1^{k}\right)$ to produce a tuple $\left(m_{0}, m_{1}, i, \beta, z\right)$.

When $\mathcal{A}$ queries $[s, W, X, Y, Z]$ to its oracle, respond as follows:

(a) Return $\perp$ if $s \neq 0$ or $Z \neq 0$, or if $W, X, Y \notin \mathbb{G}$, etc.

(b) Sample $r \in \mathbb{G}$ and use the valid re-encryption program $Z_{1} \leftarrow r^{a}, Z_{2} \leftarrow r^{b}$ and $Z_{3} \leftarrow r$ to compute a response.

4. Sample a bit $t \stackrel{r}{\leftarrow}\{0,1\}$.

5. Set $y$ to be the ciphertext $\left[0, h_{1}^{x}, h_{2}^{y}, Q \cdot m_{t}, 0\right]$ if $i=0$ and $\left[0,\left(h_{1}^{x}\right)^{a c},\left(h_{2}^{y}\right)^{b c}, Q^{c}\right.$. $\left.m_{t}, 0\right]$ if $i=1$. Furthermore, if $\beta=1$, transform $y$ into a second-form ciphertext (this can be done with public information).

6. Run $B \leftarrow \mathcal{A}_{2}^{\mathcal{O}}(y, z)$

7. Run $t^{\prime} \leftarrow D(B)$ and output 1 if $t^{\prime}=t$ (guess that $\Gamma$ is a DLA instance) and otherwise output 0 .

We argue that when $\Gamma$ is a DL instance, $\mathcal{A}^{\prime}$ perfectly simulates the experiment $\mathrm{IND}_{t}$. When $\Gamma$ is not an instance, then the encryption $y$ is independent of the message $m_{t}$ and so the probability that $t^{\prime}=t$ is exactly $1 / 2$. The proof of the theorem follows by standard probability manipulation of these two facts. 